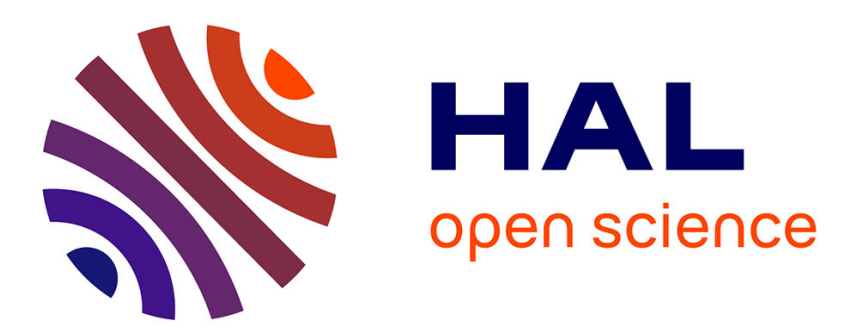

\title{
Manipulation of confined bubbles in a thin microchannel: drag and acoustic Bjerknes forces
}

David Rabaud, Pierre Thibault, Jan-Paul Raven, Olivier Hugon, Eric Lacot, Philippe Marmottant

\section{- To cite this version:}

David Rabaud, Pierre Thibault, Jan-Paul Raven, Olivier Hugon, Eric Lacot, et al.. Manipulation of confined bubbles in a thin microchannel: drag and acoustic Bjerknes forces. Physics of Fluids, 2011, 23 (4), pp.042003. 10.1063/1.3579263 . hal-00576528v2

HAL Id: hal-00576528

https://hal.science/hal-00576528v2

Submitted on 19 Apr 2011

HAL is a multi-disciplinary open access archive for the deposit and dissemination of scientific research documents, whether they are published or not. The documents may come from teaching and research institutions in France or abroad, or from public or private research centers.
L'archive ouverte pluridisciplinaire HAL, est destinée au dépôt et à la diffusion de documents scientifiques de niveau recherche, publiés ou non, émanant des établissements d'enseignement et de recherche français ou étrangers, des laboratoires publics ou privés. 


\title{
Manipulation of confined bubbles in a thin microchannel: Drag and acoustic Bjerknes forces
}

\author{
David Rabaud, Pierre Thibault, Jan-Paul Raven, Olivier Hugon, \\ Eric Lacot, and Philippe Marmottant ${ }^{\text {a) }}$ \\ UMR 5588, Laboratoire Interdisciplinaire Physique, CNRS and Université de Grenoble, \\ F-38402 Grenoble, France
}

(Received 3 September 2010; accepted 17 March 2011; published online 18 April 2011)

\begin{abstract}
Bubbles confined between the parallel walls of microchannels experience an increased drag compared to freestanding bubbles. We measure and model the additional friction from the walls, which allows the calibration of the drag force as a function of velocity. We then develop a setup to apply locally acoustic waves and demonstrate the use of acoustic forces to induce the motion of bubbles. Because of the bubble pulsation, the acoustic forces-called Bjerknes forces-are much higher than for rigid particles. We evaluate these forces from the measurement of bubble drift velocity and obtain large values of several hundreds of nanonewtons. Two applications have been developed to explore the potential of these forces: asymmetric bubble breakup to produce very well controlled bidisperse populations and intelligent switching at a bifurcation. (C) 2011 American Institute of Physics. [doi:10.1063/1.3579263]
\end{abstract}

\section{INTRODUCTION}

Recent years have seen the rise of digital microfluidics, in which tiny droplets or bubbles are handled as discrete volumes of fluids. ${ }^{1}$ We focus in the present work on microbubbles generated in flow focusing devices, bubbles characterized by very reproducible volumes., ${ }^{2,3}$ These microbubbles may act as tiny samples in lab-on-a-chip devices. ${ }^{4,5}$ Microbubbles can also act as simple spacers between droplet samples, to avoid any contact and contamination between them. ${ }^{6}$ Another application is their collection out of the microfluidic chip to obtain contrast agents ${ }^{7}$ or to fabricate new porous materials.

We further consider bubbles which diameters may exceed the height of the microchannel geometry, defined by the two parallel plates and a rectangular cross-section. Under confinement, bubbles will therefore loose their sphericity so as to adopt a shape that may be assimilated to a "french cheese" or "pancake." This geometrical confinement has to be contrasted with the confinement of bubbles inside tubes of circular cross-section, which have drawn a lot of attention on their vibrations. ${ }^{8}$

In the perspective of developing noncontact handling methods so as to avoid any contamination of the operator, the control of bubble trajectories is of major importance, and possibly with selective control. This would, for instance, allow to sort bubbles, to control reactions (thermal, chemical, biological) to perform on lab-on-chips.

We propose to take advantage of acoustic waves to manipulate bubbles. The generator of acoustic waves, a piezoelectric element, can be miniaturized and integrated in a chip. Such devices have been developed to sort solid particles ${ }^{9,10}$ and biological cells ${ }^{11}$ using acoustic radiation forces. However, the specificity of bubbles is their pulsation

${ }^{a)}$ Electronic mail: philippe.marmottant@ujf-grenoble.fr. near a resonance frequency, giving rise to Bjerknes forces $^{12-14}$ that are much larger than radiation forces on poorly compressible particles. For instance, these forces are helpful to act on bubbles rising in a liquid pool. ${ }^{15}$

In order to evaluate the efficiency of the applied acoustic forces, one can estimate the resisting drag forces. This way of reasoning is close to the one developed in the famous electrical force measurement on oil droplets by Millikan. ${ }^{16}$ However, in Millikan's experiment the droplet is in plain air and the Stokes drag formula is valid, while in the present microfluidic configuration, the bubble is confined between walls and the drag is increased by friction with the walls. Our system is therefore close to that of a bubble in a typical Hele-Shaw configuration, studied by Maruvada et al. ${ }^{17}$ The authors of this study assume a specific frictional behavior, that of a pure liquid, and do not take into account the presence of surfactants. Nevertheless, these surfactants are necessarily added in solution to prevent any undesirable bubble coalescence, when the bubble concentration is high: because surfactants can rigidify the interface, their impact has to be included in the analysis for the friction. Note that Fuerstman et al. ${ }^{18}$ have studied the pressure drop of a flowing bubble in a thin microchannel, but for the case where the bubble takes the whole width of the channel, touching the four walls of thin channels with a rectangular cross-section.

This paper is organized as follows. The materials and methods are given in Sec. II with two configurations of microfluidic channels: the first for the study of the drag force that we calibrate with the buoyancy force, and the other one for acoustic forcing. We present in Sec. III measurements of the drag force and introduce a new model for this force, which takes into account the diverse behavior of surfactants and is validated by measurements. Then we evaluate the acoustic force experienced by deviated bubbles in Sec. IV. Section $\mathrm{V}$ is devoted to the description of applications we 
(a)

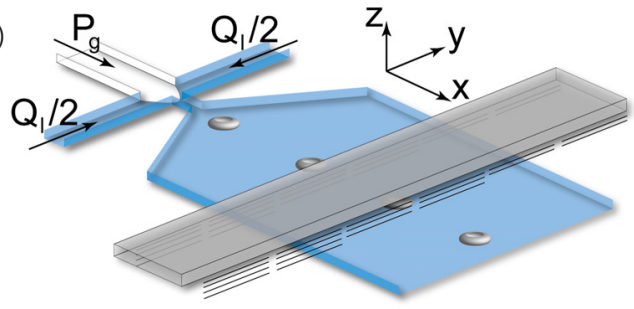

(b)

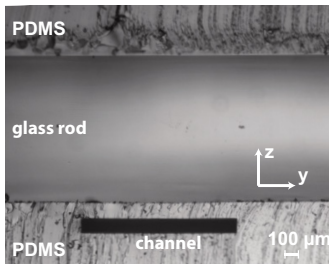

(c)

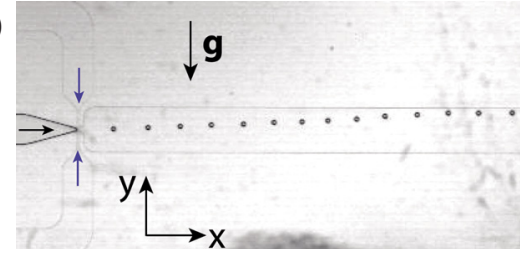

FIG. 1. (Color online) (a) Schematic geometry of an acoustically coupled microchannel: the channel is filled with water and the main bubble flow is along the $x$ direction. The channel is very thin in the $z$ direction. These axes are attached to the channel. Light gray: the glass rod, separated from the channel by a thin layer of elastomer. (b) Photograph of a cut section of the PDMS perpendicular to the microchannel $x$-axis. The position of the glass rod is above the thin channel, separated by a $100 \mu \mathrm{m}$ PDMS layer. (c) Photograph of the circuit, along $z$ direction, without the acoustic glass rod. Here it was tilted by $90^{\circ}$ around the main axis for the drag force calibration, with gravity vector along $y$-axis. For acoustic measurements, the channel was horizontal, and gravity vector along the $z$-axis.

have specifically developed: size sorting, automated switching, and asymmetric breakup at a bifurcation.

\section{THE ACOUSTOMICROFLUIDIC DEVICE}

\section{A. Experimental setup}

Acoustically operated microfluidic circuits are built using soft-lithography techniques. ${ }^{19}$ The circuit consists of two layers. The first layer includes the channel and liquid plus gas inlets which are molded in a polydimethylsiloxane (PDMS) (Sylgard 184, Dow Corning) so as to create a flow focusing geometry as shown in Fig. 1. The second layer of the circuit includes the acoustic generator consisting of a glass rod (cut from a microscope slide) molded and covered by a thin $100 \mu \mathrm{m}$ layer of PDMS. A piezoelectric ceramic (PIC151, $1 \mathrm{~mm}$ thick, Piezo-Instruments) is glued with epoxy to one end of the glass rod. The two layers are finally bonded by air plasma exposure (Harrick Plasma) with the rod perpendicular to the channel, as shown on Fig. 1. This results in a glass rod which is therefore not in direct contact with the channel, but separated by the thin $100 \mu \mathrm{m}$ layer of PDMS.

The gas thread flowing through the flow-focusing orifice is symmetrically pinched by water, creating microbubbles further dragged by the liquid. The liquid is de-ionized water with $10 \%$ commercial dishwashing detergent (Dreft, Procter and Gamble). A syringe pump (11 Pico Plus, Harvard Apparatus) is used to push the liquid in the channel. The gas (pure nitrogen) is under controlled pressure. The liquid flow rate $Q_{l}$ and the gas pressure $P_{g}$ are the control parameters for the production and the flow of the bubbles. The standard values are around $120 \mu \mathrm{m} \mathrm{min}^{-1}$ for $Q_{l}$ and $7 \mathrm{kPa}$ for $P_{g}$. The produced bubbles are flowing in channels whose thickness vary between $h=50 \mu \mathrm{m}$ and $h=100 \mu \mathrm{m}$. They are always in contact with top and bottom channel walls, and their aspect ratio varies in the range 1.3-5.2.

The bubble flow is recorded with a CMOS-camera (Marlin F131B, AVT) through an inverted microscope (IX70, Olympus). Post-treatment of images is used to extract the bubbles' characteristics such as their radii $R$ and their longitudinal and transverse velocities $U_{x}$ and $U_{y}$. The average liquid velocity $V$, along $x$, is obtained by dividing the flow rate $Q_{l}$ by the cross section of the channel: height $h$ (between 10 and $100 \mu \mathrm{m})$ times its width $w(1000 \mu \mathrm{m}$ in all our experiments).

An efficient coupling between the bubble flow and the ultrasound field is obtained by integrating the acoustic source into the microcircuit, limiting excess attenuation from the PDMS polymer matrix. The choice of a glass rod put perpendicular to the main flow is advantageous as it possesses well-defined resonance modes when excited from the piezoelectric transducer for certain frequencies. The signal sent to the transducer is produced by a function generator (AFG3102, Tektronik) and is amplified (7600M, Krohn Hite) up to $50 \mathrm{~V}$.

\section{B. Characterization of the resonance modes of the glass rod}

The resonance modes along the main axis (the $y$-axis in Fig. 1) of the glass rod are studied as they are responsible for the transverse acoustic forces on the bubbles. In order to keep a unidimensional vibration pattern, the allowed frequencies are limited to $l \leq \lambda / 2 \leq L$, where $\lambda=c / f$ is the wavelength of sound in glass, and where $l$ and $L$ are the width and length of the glass rod. For $l=3.7 \mathrm{~mm}, L$ $=25 \mathrm{~mm}$, and $c$ of the order of $1000 \mathrm{~m} \mathrm{~s}^{-1}$ [as measured, see Fig. 2(b)], the frequency is indeed contained between 20 and $140 \mathrm{kHz}$.

The acoustic pressure on the surface of the glass rod is measured by a needle hydrophone (NP10-3, Dapco). This uncalibrated hydrophone is used to detect the position of the nodes and antinodes at each frequency, rather than absolute acoustic amplitude. A sweep between 20 and $140 \mathrm{kHz}$ has been performed to identify the resonance modes. For a spatial exploration, a homemade micropositioning system with $10 \mu \mathrm{m}$ of precision has been used to perform steps of $0.1 \mathrm{~mm}$ along the $y$-axis. The lubricated needle is in contact with a free glass rod, not molded in the PDMS matrix.

We measure the wave number $k$ of the vibration patterns [Fig. 2(a)] along the $y$-axis, taking into account the fact that the wavelength of the pattern is half of the vibration wavelength, because the needle hydrophone gives only the absolute value of the acoustic signal. We have access to the speed of the standing wave through the relation $c=\omega / k$, and we 
(a)

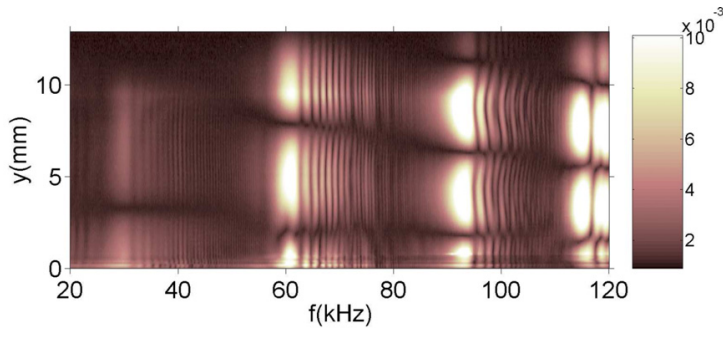

(b)

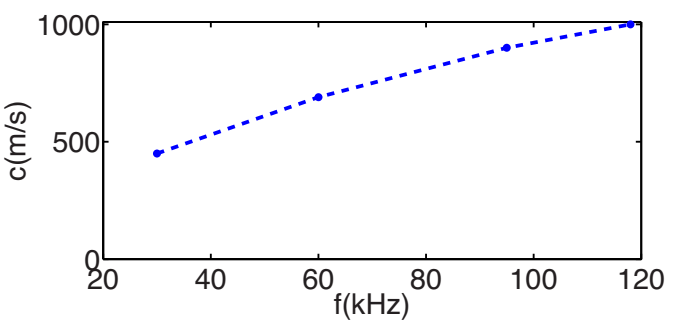

(c)

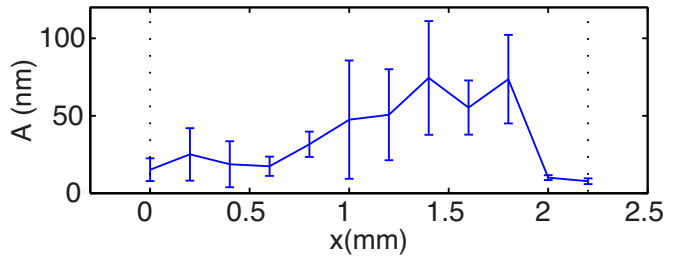

FIG. 2. (Color online) Vibration of the free glass rod, before molding in PDMS: (a) spatial-frequency diagram of the resonating rod. $y=0$ corresponds to the free end, the forced end (the piezoelectric) is approximately at $y=25 \mathrm{~mm}$. The color bar indicates the zero to peak voltage values measured on the hydrophone. (b) Wave speed obtained with the spatial Fourier transform, same scale of the abscisses as in (a); (c) internal vibration of the top channel wall, after molding and assembly of the circuit, measured just below the glass rod whose limits are shown by dotted lines, along the channel $(x$-axis). Each point is an average of the vibration in the width of the channel $(f=62 \mathrm{kHz})$.

find that $c$ varies from 450 to $1000 \mathrm{~m} \mathrm{~s}^{-1}$ between 20 and $120 \mathrm{kHz}$ [Fig. 2(b)]. These values are typical of the first antisymmetric Lamb waves, in agreement with the work of Haake. ${ }^{10}$ The vibrations emitted by the glass rod propagate to the channel and liquid, the PDMS attenuation being small. We measured an attenuation of longitudinal waves in bulk PDMS of $7 \mathrm{~dB} \mathrm{~cm}{ }^{-1} \mathrm{MHz}^{-1},{ }^{20}$ meaning an attenuation by propagation of $0.007 \mathrm{~dB}$ (through $100 \mu \mathrm{m}$ at $100 \mathrm{kHz}$ ), much smaller than the transmission coefficient at the glass/ PDMS and PDMS/water interfaces. The wavevector for the vibration of the liquid in the channel is therefore $k=k_{\mathrm{Lamb}}$, and thus slightly different from the wavevector in water $k_{w}$.

We measured the vibration of the channel wall in situ, with a distant vibrometer based on a LOFI system (Laser Optical Feedback Interferometer), ${ }^{21}$ in order to check that the vibrations are localized near the glass rod. The system, based on an autodyne interferometer, is particularly efficient and easy to implement. Its main features are that it is self-aligned and extremely sensitive, thanks to a signal resonance amplification phenomenon. For this study, the setup was composed of a Nd:YAG (neodymium-doped yttrium aluminum garnet) microlaser whose beam was frequency shifted by acoustooptic modulators and then injected into a microscope. When the beam is focused on a scattering interface, we let the backscattered light be reinjected into the laser, causing its

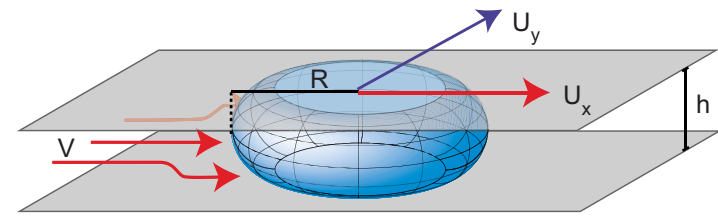

FIG. 3. (Color online) Sketch of the shape of the bubbles confined by the upper and lower faces of the channel.

intensity to be modulated at the shift frequency. If the interface under investigation is vibrating, there is a modulation of this frequency by the Doppler effect, generating sidebands around the shift frequency in the laser power spectrum. The vibration amplitude of the interface is then gathered from those sideband amplitude using a Bessel analysis.

The results obtained for the amplitude of vibration of the channel (here filled with air) just below the glass rod are summarized in Fig. 2(c). The vibration amplitude is much attenuated out of the borders of the glass rods. In conclusion, the vibration is localized in the channel, and the variation in acoustic amplitude follows that of the glass rod.

\section{DRAG FORCE FOR A CONFINED BUBBLE IN HELE-SHAW CONFIGURATION}

\section{A. Bubble shape in the surfactant solution}

The shape of a bubble confined in a microchannel is illustrated in Fig. 3: near the top and bottom channel walls, it possesses almost flat faces lubricated by thin liquid films, while the side surface is curved. Because of the presence of surfactant molecules in water, the wetting of the channel surfaces is always satisfactory, meaning a vanishing contact angle. The shape of the bubble is parametrized by the channel thickness $h$, and the outer radius $R$, easy to measure on the image of the bubble when projected perpendicularly to the planes.

\section{B. Measurements under flow and transverse applied force}

The drag force $\mathbf{F}_{\text {drag }}$ in such a confined geometry differs notably from the Stokes expression for a sphere in the bulk of a fluid $\mathbf{F}_{\text {Stokes }}=6 \pi \mu R(\mathbf{V}-\mathbf{U})$, where $\mu$ is the dynamic viscosity of the liquid, $R$ the radius of the bubble, $\mathbf{V}$ the average speed of the fluid, and $\mathbf{U}$ the bubble speed.

We measured the bubble velocity in a fluid stream $V$ under a transverse applied force $F_{y}$. To obtain a controlled applied force, we just used the buoyancy force: in the absence of ultrasound, the circuit is inclined with a variable angle $\theta$ (from $0^{\circ}$ to $90^{\circ}$ ) around the $x$-axis. The gravity vector $\mathbf{g}$ being perpendicular to the flow [see Fig. 1(b)], the bubbles undergo the buoyancy force and are pulled up in the transverse direction. This external force is given by $F_{\text {applied, } y}=\Delta \rho \pi R^{2} h g \sin \theta$, where $\Delta \rho$ is the difference of density between air and water, and where we assume cylindrical bubbles as an approximation of their real shape, shown in Fig. 3. Due to the low Reynolds number (between 0.1 and 1), a steady state of the velocity is quickly reached so that 


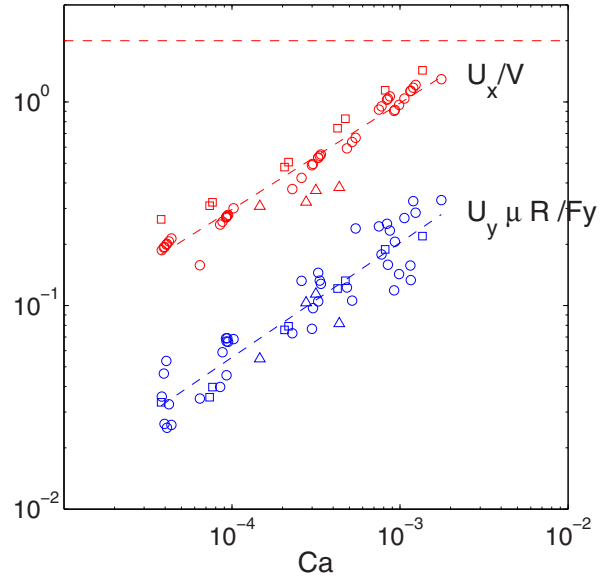

FIG. 4. (Color online) Measurements of the axial bubble velocity $U_{x}$ induced by the stream velocity $V$ and deviation velocity $U_{y}$ induced by a transverse force $F_{y}$, as a function of the capillary number based on the norm of the bubble velocity. Dashes: Saffman-Taylor model for a bubble in pure water, which neglects wall friction $\left(a_{\text {wall }}=0\right)$. Several channel thicknesses are tested: $\triangle, h=50 \mu \mathrm{m} ; \bigcirc, 70 \mu \mathrm{m} ; \square, 100 \mu \mathrm{m}$. The bubble radii vary from 40 to $80 \mu \mathrm{m}$ for all thicknesses. Power-law fits are plotted as dashed lines, with exponents of 0.52 for the scaled $U_{x}$ and 0.56 for the scaled $U_{y}$.

$\mathbf{F}_{\text {drag }}+\mathbf{F}_{\text {applied }}=0$. The drag force components are therefore $F_{\text {drag }, x}=0$ and $F_{\text {drag }, y}=-F_{y}$. For a Stokes drag we would thus obtain $U_{x}=V$ and $U_{y}=F_{y} / 6 \pi \mu R$.

The measurements displayed in Fig. 4 show that this is not the case: $U_{x} / V$ and $U_{y} / F_{y} \mu R$ are not the expected constants 1 and $1 / 6 \pi$, but depend on the bubble velocity. Here we have nondimensionalized the bubble velocity using

$$
\mathrm{Ca}=\frac{\mu U}{\sigma}
$$

the capillary number, constructed with the norm of the bubble velocity $U=\left(U_{x}^{2}+U_{y}^{2}\right)^{1 / 2}$ and the surface tension $\sigma$ (measured to be approximately equal to $35 \times 10^{-3} \mathrm{~N} \mathrm{~m}^{-1}$ for our surfactant solutions). A first phenomenological description of the effect of the capillary number is to fit the measured values with power laws, which is shown on Fig. 4. The effect of bubble size and of channel height will be treated in the following section.

\section{Model for the drag force}

In the present configuration, we model the drag force as the sum of a viscous drag (of the liquid on the bubbles) and a friction force (between the bubbles and the walls of the channel), so that the total drag is

$$
\mathbf{F}_{\text {drag }}=a_{\text {fluid }} \frac{12 \pi \mu R^{2}}{h}(2 \mathbf{V}-\mathbf{U})-a_{\text {wall }} \mu h\left[\left(\frac{h}{R}\right)^{3} \mathrm{Ca}\right]^{\alpha-1} \mathbf{U},
$$

where $h$ is the height of the channel and $\mathrm{Ca}=\mu U / \sigma$ is the capillary number based on the norm of the bubble velocity vector.

The first term of the right-hand side is the friction from the bulk fluid. This equation has been used by Maruvada $e t$ $a l .,{ }^{17}$ who derived an expression for the Stokes drag for a cylindrical bubble, with slip conditions, following the analy- (a)

(b)

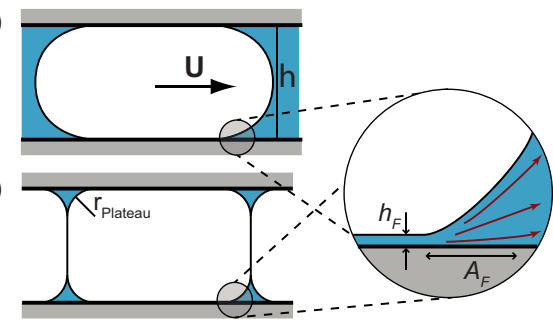

FIG. 5. (Color online) Cross-section of (a) one isolated bubble; in white the gas. (b) Comparison with a foam composed of one layer of bubbles. The insert is an enlargement on the liquid corners where dissipation is localized in both cases.

sis of Taylor and Saffman in Hele-Shaw configuration. ${ }^{22}$ We add a free parameter $a_{\text {fluid }}$ to account for a deviation from a purely cylindrical bubble and to account for the influence of surfactants that would change the slip conditions.

The second term of the right-hand side, the friction with the walls, is a correction to the formula ${ }^{17}$ and extends it for the presence of surfactants. This presence modifies the variation of the friction forces with velocity. We introduce phenomenological coefficients $a_{\text {wall }}$ and $\alpha$, depending on the nature of the surfactant. In the following we present a derivation of the equation we use for this friction force with the walls.

Such a phenomenological approach is classical in slipping foam studies with different types of surfactants, but here we feel it is necessary to recall its foundations. First, we introduce the phenomenological exponent $\alpha$. A dimensional analysis shows that the friction force scales like $F_{\text {wall }}$ $\sim \mu\left(U / h_{F}\right) A_{F},{ }^{23}$ where $h_{F}$ is the film thickness and $A_{F}$ is the area of friction (see Fig. 5). In the case of a free interface (pure water) or an interface with very mobile surfactant molecules, the Bretherton theory ${ }^{24}$ states that the film thickness increases with bubble velocity ("aquaplaning" effect or Landau-Levich lubrication) so that $h_{F} \sim h \mathrm{Ca}^{2 / 3}$, while the friction area varies like $A_{F} \sim h^{2} \mathrm{Ca}^{1 / 3}$. The friction force therefore may be expressed as $F_{\text {wall }} \sim \mu h \mathrm{Ca}^{-1 / 3} U$ (and is thus proportional to $U^{2 / 3}$ ). This is in contradiction to the analysis of Maruvada et al. ${ }^{17}$ where the friction area $A_{F}$ was incorrectly set to be a constant (this yields a force proportional to $\left.U^{1 / 3}\right)$. In the case of immobile surfactants, the surface is rigid, and it has been shown ${ }^{23}$ that $h_{F} \sim h \mathrm{Ca}^{1 / 2}$, while the friction area is constant, so that $F_{\text {wall }} \sim \mu h \mathrm{Ca}^{-1 / 2} U \sim U^{1 / 2}$. See Table I for a summary.

These two cases, mobile and immobile surfactants, can be extrapolated to any type of surfactants by postulating that the force may be expressed as $F_{\text {wall }} \sim \mu h \mathrm{Ca}^{\alpha-1} U$ (force proportional to $U^{\alpha}$ ), the value of $\alpha$, between $1 / 2$ and $2 / 3$, reflect-

TABLE I. Summary of the friction models with mobile and immobile surfactants.

\begin{tabular}{lccc}
\hline \hline Surfactant & Film thickness & Friction area & Friction force \\
\hline Mobile $^{\mathrm{a}}$ & $h_{F} \sim h \mathrm{Ca}^{2 / 3}$ & $A_{F} \sim h^{2} \mathrm{Ca}^{1 / 3}$ & $F_{\text {wall }} \sim \mu h \mathrm{Ca}^{-1 / 3} U$ \\
Immobile $^{\mathrm{b}}$ & $h_{F} \sim h \mathrm{Ca}^{1 / 2}$ & $A_{F} \sim \mathrm{cst}$ & $F_{\text {wall }} \sim \mu h \mathrm{Ca}^{-1 / 2} U$ \\
\hline \hline
\end{tabular}

${ }^{\mathrm{a}}$ Reference 24.

${ }^{\mathrm{b}}$ Reference 23. 


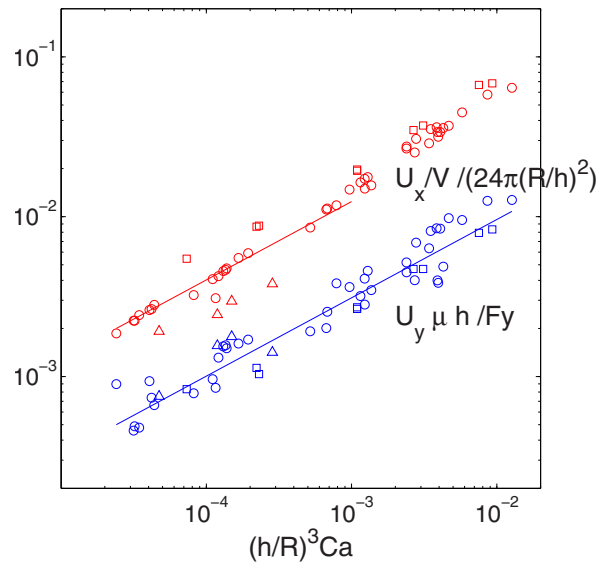

FIG. 6. (Color online) Adimensioned velocity components $U_{x}$ (top) and $U_{y}$ (bottom), as a function of $(h / R)^{3} \mathrm{Ca}$, proportional to the total bubble velocity $U$. Lines: power fits with Eqs. (2) and (3) are $\alpha=0.51, a_{\text {wall }}=10.9$, and $a_{\text {fluid }}=4.0$. Only the values $U_{x} / V<0.5$ are fitted. Same symbols than in Fig. 4.

ing the mobility of the surfactant. This variable exponent is widely used in slipping foam studies, ${ }^{25}$ as well as in foam drainage or rheology characterization. ${ }^{26}$

Second, we have also introduced the influence of the ratio of the thickness to the bubble size $h / R$. We are inspired by the predictions of Terriac et $a l^{27}$ for bubbles in contact (thus forming a foam) in Hele-Shaw cells. They consider that dissipation is actually localized within liquid corners, and this is the radius of the liquid corners that counts (see inset of Fig. 5). In the case of foams, the liquid corner as a radius equal to the radius of the Plateau border, i.e., the meniscus joining liquid to walls [see Fig. 5(b) for the definition of $\left.r_{\text {Plateau }}\right]$. Here the liquid corner has a radius equal to half of the channel height $r_{\text {Plateau }}=h / 2$. Equivalently, the bubbles in a foam have the same shape as isolated bubbles when $r_{\text {Plateau }}=h / 2$. Setting this value for the liquid corner size, the analysis of Terriac for a bubble of spanwise diameter $2 R$ (since the spanwise width of the liquid corner at the walls sets the total friction ${ }^{28}$ ) yields a friction force $F_{\text {wall }}$ $\sim 2 R \sigma(h / R)^{-2}\left[(h / R)^{3} \mathrm{Ca}\right]^{\alpha} \sim \mu h\left[(h / R)^{3} \mathrm{Ca}\right]^{\alpha-1} U$, that we use in Eq. (1).

In conclusion, the total drag force is given by Eq. (1), in which the phenomenological coefficients $\left(\alpha, a_{\text {fluid }}\right.$, and $\left.a_{\text {wall }}\right)$ will be adjusted in experiments.

The projections of Eq. (1) onto the $x$ - and $y$-axes give the bubble velocity components $U_{x}$ and $U_{y}$ as a function of the external flow $V$ (here along the $x$-axis) and transverse force $F_{\text {applied, } y}$ (along the $y$-axis),

$$
\begin{aligned}
& \frac{U_{x}}{V} \simeq 24 \pi\left(\frac{R}{h}\right)^{2} \frac{a_{\text {fluid }}}{a_{\text {wall }}}\left[\left(\frac{h}{R}\right)^{3} \mathrm{Ca}\right]^{1-\alpha}, \\
& \frac{\mu h U_{y}}{F_{\text {applied, } y}}=\frac{1}{a_{\text {wall }}}\left[\left(\frac{h}{R}\right)^{3} \mathrm{Ca}\right]^{1-\alpha} .
\end{aligned}
$$

To obtain Eq. (2), we have assumed $U_{x} \ll V$.

We plot in Fig. 6 the measured bubble velocity components $U_{x}$ and $U_{y}$ scaled by $V \times 24 \pi(R / h)^{2}$ and $F_{\text {applied,y }} / \mu h$, respectively, versus $(h / R)^{3} \mathrm{Ca}$. All the points collapse on power law curves as expected from Eqs. (2) and (3), for a wide range of conditions: $h$ ranges from 50 to $100 \mu \mathrm{m}, R$ from 34 to $83 \mu \mathrm{m}$, and $V$ from 7 to $33 \mathrm{~mm} \mathrm{~s}^{-1}$. With this new rescaling, data points are slightly less dispersed than in Fig. 4. The dispersion in the values of the scaled $U_{y}$ drops from $\pm 29 \%$ down to $\pm 27 \%$. Additionally, the range of the scaled data is larger by one order of magnitude. We therefore use this new scaling designed to describe large range of variations in channel thickness and bubble radius. So we are able to adjust the parameters $\alpha, a_{\text {wall }}$, and $a_{\text {fluid }}$ in Eqs. (2) and (3).

We now discuss the value of $\alpha$, the exponent of the Capillary number in Eq. (3). According to Denkov et al., ${ }^{23}$ this exponent reflects the nature of the tangential mobility of the surfactant molecules on the surface of the bubbles. The value $\alpha=0.51$ corresponds to tangentially immobile surfactants. This may be due to large Marangoni stresses. Here the concentration of surfactant in the solution is high, 40 times the critical micellar concentration, and the surface tension drops down to $\sigma=35 \times 10^{-3} \mathrm{~N} / \mathrm{m}$, instead of $\sigma_{w}=73$ $\times 10^{-3} \mathrm{~N} / \mathrm{m}$ for a clean interface. Surfactants can therefore create Marangoni surface stresses up to $\Delta \sigma=\sigma_{w}-\sigma=38$ $\times 10^{-3} \mathrm{~N} / \mathrm{m}$. The ratio of these stresses to viscous stresses may be expressed as $\Delta \sigma / \mu U=\Delta \sigma / \sigma \times \mathrm{Ca}^{-1}$. In the present experiment this ratio is always above 500 (value at largest velocity), meaning that surfactants create surface stresses that are high enough to immobilize the surface in front of viscous stresses.

This phenomenon is important because it governs the nature of the friction on the walls of the channel. The value of the coefficient $a_{\text {fluid }}=4.53$, that gives the friction from the flow around the bubble see Eq. (1), is substantially higher than 1, which also suggests that instead of a slip velocity there is an immobile velocity at the surface.

In conclusion, we have calibrated the friction force acting on confined bubbles. We are thus able to deduce the value of any transverse external force acting on the bubbles from the measurement of their drift velocity (any external force is equal and opposite to the transverse drag). According to Eq. (1) and from several calibrations, we end up with an empirical formula that may be expressed as $F_{\text {applied, } y}$ $\simeq 10.9 \mu h\left[(h / R)^{3} \mathrm{Ca}\right]^{-0.49} U_{y}$.

\section{THE ACOUSTIC BJERKNES FORCE FOR A CONFINED BUBBLE}

\section{A. Measurements from deviation velocity in the acoustic field}

A measurement of acoustic force applied to the bubbles is obtained when considering the acoustic deviation velocity $U_{y}$ of the bubbles in the absence of gravity effects (horizontal channel) and during the emission of an ultrasonic standing wave.

We plot in Fig. 7 the results obtained for the acoustic force as a function of the bubble radius under constant acoustic conditions (frequency of $91 \mathrm{kHz}$, amplitude of the electric signal sent to the piezo-50 V). The first striking feature is the large amplitude of the acoustic forces: they amount to nearly $200 \mathrm{nN}$, enough to counteract the friction forces that are high 


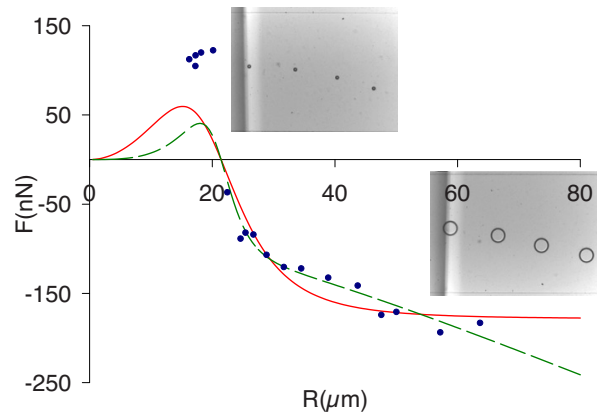

FIG. 7. (Color online) Bjerknes force vs the radius of the bubbles at fixed frequency (91 kHz); dots: experimental data; dashes: Eq. (14); line: Eq. (15); channel height $h=25 \mu \mathrm{m}$. Inserts: pictures of bubbles with radii of 17 (top) and 63 (bottom) $\mu \mathrm{m}$.

in this geometry. The second feature we notice is that the sign of the force changes with the size, with a critical radius.

\section{B. Modeling the bubble pulsation in a confined channel}

To interpret these results, we are going to first model the pulsation of the bubble. Indeed the compressible volume of gas oscillates in response to the oscillating acoustic pressure. For small acoustic amplitudes, the response is linear and described by the same equation as that of a forced mechanical mass-spring oscillator. The surrounding fluid displaced during the pulsation plays the role of a mass, while the compressibility of the gas plays the role of a spring.

The Minnaert formula ${ }^{13}$ gives the resonance frequency for spherical air bubbles in water,

$$
f_{r}^{3 \mathrm{D}}=\frac{1}{2 \pi} \sqrt{\frac{3 \kappa P_{0}}{\rho}} \frac{1}{R},
$$

with $\kappa$ the gas polytropic exponent, $P_{0}$ the pressure in the liquid, and $\rho$ the liquid density. This gives the approximate relation $f_{r} \cdot R \simeq 3 \mathrm{~m} \mathrm{~s}^{-1}$ at resonance. In our experiments, the bubbles are confined and pulse in a different manner. Prosperetti ${ }^{29}$ gave the relation between the resonance frequency and the size of a purely cylindrical bubble,

$$
f_{r}^{2 \mathrm{D}}=\frac{1}{2 \pi} \sqrt{\frac{2 \kappa P_{0}}{\log (S / R) \rho}} \frac{1}{R},
$$

with $S$ the radius of a finite cylindrical region occupied by the fluid. This length could be interpreted as a cutoff length associated with the finite channel size, channel elasticity, or fluid compressibility. Here, fluid compressibility of water (with a bulk modulus of $2.2 \mathrm{GPa}$ ) can be neglected when compared to the PDMS channel elasticity [with Young's modulus of the order of $1 \mathrm{MPa}$ (Ref. 30)]. Because the acoustic pressure levels are fractions of $1 \mathrm{kPa}$ (see further), we expect that side boundaries, as well as top and bottom boundaries, are deformed during the bubble oscillation. As a consequence, the closest side boundaries, situated at half the channel width, do not completely impede the motion. We have therefore chosen for the length $S$ the channel halfwidth, considering that it is the furthest distance where fluid inertia is present when channels are very soft. The channel width is $1000 \mu \mathrm{m}$ in all our experiments, and bubbles of radius of $20 \mu \mathrm{m}$, we obtain $f_{r}^{2 \mathrm{D}} \cdot R \approx 1 \cdot 3 \mathrm{~m} \mathrm{~s}^{-1}$ and slightly more for larger bubbles.

For the present work, we impose a single value of $K$ $=f_{r} \cdot R$ for the whole range of diameters explored, that lies between the two-dimensional (2D) and three-dimensional (3D) case, so that

$$
f_{r}^{\text {confined }}=K \frac{1}{R} .
$$

We define the oscillation of the pressure in a standing wave as

$$
p=P_{0}+P_{A} \sin (k y) \cos (\omega t),
$$

where $f=\omega / 2 \pi$ is the frequency of oscillation and $k$ is the wave vector. The two cases of confined bubbles (assumed cylindrical for simplicity) and free bubbles (spherical) are investigated here to show the effect of the confinement. We consider that the bubble radius and volume vary according to

$$
\begin{aligned}
& R=R_{0}[1-\varepsilon \cos (\omega t-\varphi)], \\
& \mathcal{V}=\mathcal{V}_{0}[1-n \varepsilon \cos (\omega t-\varphi)],
\end{aligned}
$$

where $\varepsilon(t) \ll 1$ and $n$ is equal to 2 or 3 for cylindrical or spherical bubbles, with respective initial volumes $\mathcal{V}_{0, n=2}$ $\simeq \pi R_{0}^{2} h$ and $\mathcal{V}_{0, n=3}=4 / 3 \times \pi R_{0}^{3}, R_{0}$ being the rest radius in both cases.

The relative oscillation amplitude $\varepsilon$ can be obtained by solving the linearized version of the Rayleigh-Plesset equation, ${ }^{29}$ that falls into the one of a forced harmonic oscillator. We obtain for the amplitude of the oscillation

$$
\varepsilon=\frac{P_{A} \sin (k y)}{\rho R^{2}} \frac{1}{\sqrt{\left(\omega_{r}^{2}-\omega^{2}\right)^{2}+\left(\omega \omega_{r} / Q\right)^{2}}},
$$

where $Q$ is the quality factor of the oscillator. We have assumed a constant confinement such that the prefactor present in the original formula of Ref. 29 is $(n-2) /(1-R / S)^{n-2} \simeq 1$. The confinement has no effect on the phase difference, which obeys

$$
\cos (\varphi)=\frac{\omega_{r}^{2}-\omega^{2}}{\sqrt{\left(\omega_{r}^{2}-\omega^{2}\right)^{2}+\left(\omega \omega_{r} / Q\right)^{2}}} .
$$

\section{Bjerknes force on a compressible bubble}

The origin of the deviation force is the Bjerknes force, which occurs when the volume of the object pulsates under ultrasound. Because of this volume pulsation $\mathcal{V}(t)$, the oscillating acoustic pressure can have a nonvanishing effect on average. The pressure force on an object which is small compared to the wavelength may be expressed as $\int_{S}-p \mathbf{n} d S=$ $-\mathcal{V} \nabla p$, with the pressure gradient $\nabla p$. The Bjerknes force is the average of this oscillating pressure force, ${ }^{14}$

$$
F_{\text {Bjerknes }}=\langle-\mathcal{V}(t) \nabla p(t)\rangle,
$$

where $\langle\cdot\rangle$ is the time average over one cycle of oscillation, which does not vanish because $\mathcal{V}$ oscillates for a bubble, unlike a rigid particle. 
Using Eqs. (7) and (9) in Eq. (12) and calculating the time average, we obtain an expression for the Bjerknes force,

$$
F_{\text {Bjerknes }}=n \varepsilon \mathcal{V}_{0}(\nabla p)_{m} \frac{1}{2} \cos (\varphi) \text {. }
$$

This expression shows that the Bjerknes force is directly proportional to the cosine of the phase difference. It is well known that any oscillator responds with a phase difference of 0 when it is excited with a frequency smaller than its resonance frequency, and a phase difference equal to $\pi$ for larger excitation frequencies. According to that, the Bjerknes force can change its sign with this phase difference.

Keeping the excitation frequency constant, we see that a resonance radius $R_{r}$ exists, obeying $f \cdot R_{r}=f_{r} \cdot R$. From this relation we expect that bubbles smaller than this resonance radius are below resonance, i.e., they oscillate with a frequency below their resonance frequency and thus have a phase difference of 0 with excitation. Therefore, the Bjerknes force in Eq. (13) will be positive (toward high acoustic pressure) for bubbles smaller than the resonance radius, and negative (toward low acoustic pressure) for bubbles bigger than the resonance radius.

Introducing the microfluidic device geometry depicted in Fig. 1 (see Sec. II, for details), we consider a channel oriented along $x$ and a transverse standing wave along $y$ created by a glass rod, placed slightly above the channel, and driven to resonance by a piezoelectric transducer at a frequency $f$. Assuming the glass rod vibration is transmitted into the channel, bubbles flowing underneath the glass rod experience the spatially modulated acoustic field $p(y, t)=P_{0}$ $+P_{A} \sin (k y) \cos (\omega t)$, with $k$ the wavenumber of the standing wave along the glass rod with velocity $c$. Note that this wavenumber characterizing the extended acoustic source differs from that of the acoustic wave propagating into water, which is given by $k_{w}=\omega / c_{w}$, with $c_{w}$ the sound velocity in water.

Finally, we are able to find an expression of the Bjerknes force, writing the pressure gradient amplitude as $(\nabla p)_{m}$ $=k P_{A} \cos (k y)$ and using Eq. (10) in Eq. (13),

$$
\begin{aligned}
F_{\text {Bjerknes }}^{2 \mathrm{D}}= & 2 \pi k h \frac{1}{k_{w}^{2}} E_{A}^{2 \mathrm{D}} \sin (2 k y) \\
& \times \frac{\left(\omega_{r} / \omega\right)^{2}-1}{\left[\left(\omega_{r} / \omega\right)^{2}-1\right]^{2}+\left(\omega_{r} / Q \omega\right)^{2}}, \\
F_{\text {Bjerknes }}^{3 \mathrm{D}}= & 4 \pi k R \frac{1}{k_{w}^{2}} E_{A}^{3 \mathrm{D}} \sin (2 k y) \\
& \times \frac{\left(\omega_{r} / \omega\right)^{2}-1}{\left[\left(\omega_{r} / \omega\right)^{2}-1\right]^{2}+\left(\omega_{r} / Q \omega\right)^{2}} .
\end{aligned}
$$

We have placed in evidence the acoustic energy density $E_{A}$ $=P_{A}^{2} / 4 \rho c_{w}^{2}$ of the standing wave, composed of two counterpropagating waves of energy $E_{A}^{\prime}=\left(P_{A} / 2\right)^{2} / 2 \rho c_{w}^{2}$. These expressions are similar except for one difference: the prefactor of the first one depends linearly on the height of the channel $h$, whereas the other depends linearly on the radius of the bubble $R$.

We now evaluate the order of magnitude of these forces in the 3D case. This acoustic energy has a simple meaning:
$4 E_{A}$ is the radiation pressure exerted on a totally reflecting surface by a propagating wave. For a comparison, we write the acoustic force for a bubble and a rigid particle computed by King, ${ }^{31}$

$$
\begin{aligned}
& \frac{F_{\mathrm{Bjerknes}}^{\max }}{\pi R^{2}} \sim \frac{4 Q}{k_{w} R} \frac{k}{k_{w}} E_{A} \sim 500 E_{A}, \\
& \frac{F_{\mathrm{King}}}{\pi R^{2}} \sim k_{w} R E_{A} \sim 0.01 E_{A},
\end{aligned}
$$

where we have evaluated these orders of magnitude by considering a bubble in water at resonance, the 3D Minnaert formula implies $k_{w} R=2 \pi f_{r} R / c_{w} \simeq 0.012$, typical values of $k / k_{w}=c_{w} / c \simeq 1.5$ and $\varepsilon \cos (\varphi) \sim Q \simeq 1$. The acoustic force on a solid particle of the same size is much less than with a reflecting surface, and more than four orders of magnitude less than the Bjerknes force.

\section{Comparison with the measurements}

The fit of force measurements with the 2D model [Eq. (14)] and with the 3D model [Eq. (15)] leads to parameters that are $Q^{2 \mathrm{D}}=1$ and $Q^{3 \mathrm{D}}=2$ [for comparison, the quality factor of a free spherical bubble of $25 \mu \mathrm{m}$ radius is around 8 (Ref. 14)] with acoustic amplitudes $E_{A}^{2 \mathrm{D}}=0.43 \mathrm{~J} \mathrm{~m}^{-3}$ and $E_{A}^{3 \mathrm{D}}=0.086 \mathrm{~J} \mathrm{~m}^{-3}$ if we assume the channel is located on an antinode of the acoustic force [i.e., $\sin (2 k y)=1$ ]. These acoustic amplitudes correspond to pressure amplitudes $P_{A}^{2 \mathrm{D}}$ $=0.62$ bar and $P_{A}^{3 \mathrm{D}}=0.28$ bar.

The overall agreement indicates that both models provide a good phenomenology of the acoustic forcing, the bubbles being neither spherical nor cylindrical but in between, as sketched in Fig. 3. Whatever the exact shape is, the sign of the force depends on the size: the bubbles with a radius greater than the resonance radius $R_{r}$ are attracted by the nodes, as opposed to the smaller ones, which are attracted to the antinodes. This is well expected by the model in Sec. IV. The resonance radius (here $21 \mu \mathrm{m}$ ) gives a Minnaert relation $f R_{r}=K$, with a value of $K=1.95 \mathrm{~m} \mathrm{~s}^{-1}$ that lies between the values for a " $2 \mathrm{D}$ " pulsation $\left(K \simeq 1.3 \mathrm{~m} \mathrm{~s}^{-1}\right)$ and a "3D" pulsation $\left(K=3 \mathrm{~m} \mathrm{~s}^{-1}\right)$, as explained in Sec. IV.

\section{APPLICATIONS OF ACOUSTIC MANIPULATION}

\section{A. An efficient size sorting device}

The inserts in Fig. 7 already illustrate the possibility of using the Bjerknes force to separate polydisperse bubbles into two subcategories by an appropriate choice of the central frequency, corresponding to a resonance size. Bubbles whose radii are 20 and $22.5 \mu \mathrm{m}$ are subjected to forces in opposite directions, highlighting the strong size selectivity of this method.

In practice, the situation is not so simple for an assembly of bubbles. The first drawback is that the amplitude of the force itself is size dependent. The amplitude of the force on these bubbles, of 20 and $22.5 \mu \mathrm{m}$ of radius, varies by a factor of 3 , leading to a distance of deviation of same ampli- 
(a)

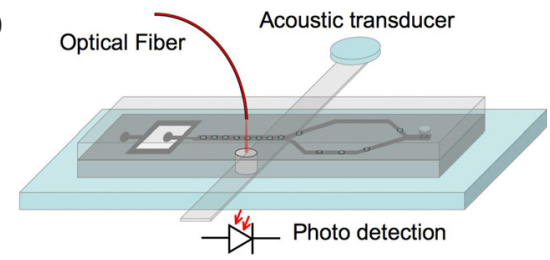

(b)

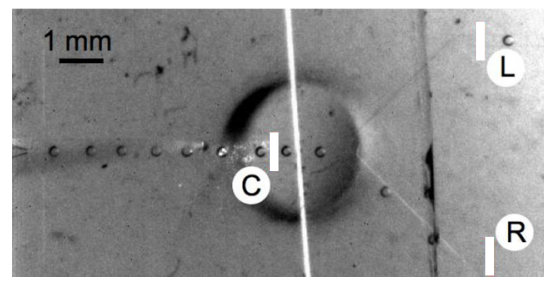

FIG. 8. (Color online) (a) Scheme of the bubble switching experiment showing the optical detection system and acoustic actuation. A $4.5 \mu \mathrm{m}$ monomode optical fiber is directly plugged into the microfluidic circuit. Light scattered from bubbles is detected on a $p-i-n$ photodiode. (b) Top view of bubbles flowing in the microchannel with a $\mathrm{Y}$ junction. Bubbles are emitted from the focusing device at the left side of the picture. The bubble emission frequency is optically measured in the central $\mathrm{C}$ branch, their passing time in each branch is recorded at the white marks $\mathrm{L}$ and $\mathrm{R}$.

tude three times longer. The second is that the bubbles may also be coupled by multibubble effects (hydrodynamic coupling, secondary acoustic forces).

\section{B. Programmable switching of bubbles}

In order to prefigure bubble manipulation in a lab-onchip, we have tested the two elementary operations of bubble detection and forcing. Bubbles are detected when they pass through a laser beam, by means of an optical fiber coming from above the setup. The signal, detected with a photodiode, is acquired on a computer and analyzed on the fly. The computer triggers a signal processing card in order to generate the appropriate acoustic signal.

Bubble switching by acoustic means was tested on a Y junction geometry, as illustrated in Fig. 8. The switching is activated by using the appropriate acoustic signal using two different ultrasound frequencies of the same amplitude, and therefore selecting two different positions for the nodes that attract the bubbles, either to the right or to the left. The signal consists of a sequence of three left-branch and three rightbranch instructions. These instructions comprise not only a frequency but also a delay that corresponds to the time it takes for each bubble to reach the bifurcation and an activation time that is set by the minimum time required for a bubble to travel across the half width of the channel.

Figure 9(a) shows the histogram of time intervals between successive bubbles passing through the laser detection beam. It can be seen that the periodicity of bubble generation is widely distributed around $T_{1}=67 \mathrm{~ms}$, ranging from 50 to $100 \mathrm{~ms}$. This is characteristic of our flow focusing device where the applied pressure gradient for air couples with the pressure drop due to the bubbles already present in the circuit. The presence of peaks at $T_{2} \simeq 4 T_{1}$ in Fig. 9 in each branch is the signature of the efficient switching: they correspond to the blank interval when bubbles are switched to the other branch, see Fig. 9(b). In this limit case of efficiency, (a)

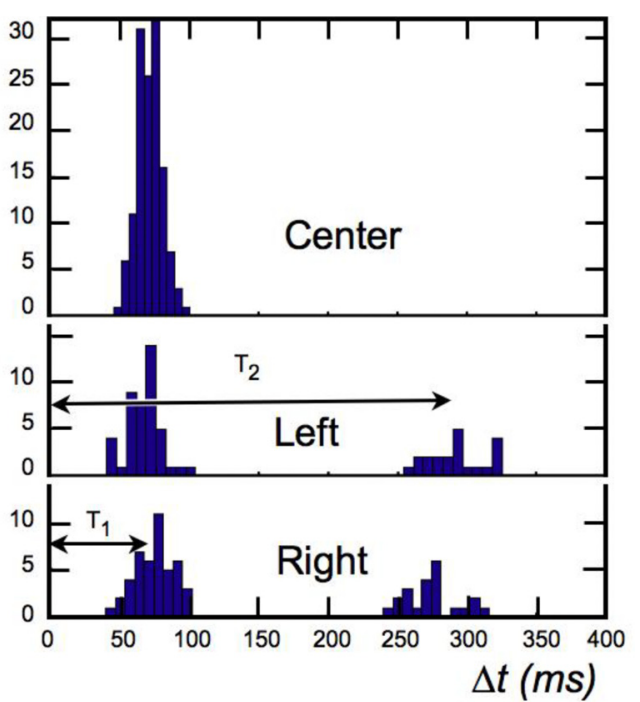

(b)

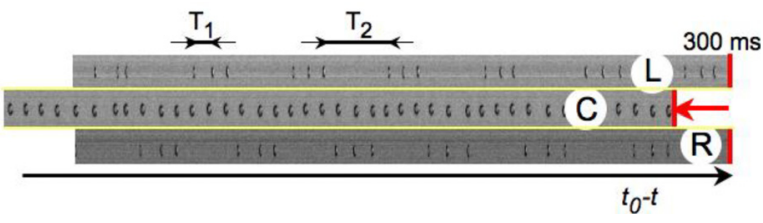

FIG. 9. (Color online) (a) Histogram of the time intervals between two successive bubbles, at different positions in the device shown in the previous figure (labels L,C,R). (b) Chronogram obtained by the horizontal juxtaposition of vertical lines of pixels (these pixel lines are L,C,R in the previous figure) at different times. Here in reverse order to show the spatial ordering. Because the bubble velocity $U_{x}$ is constant, the plot is only a function of $u=x / U_{x}-t$. The present temporal axis $u(t)=x_{0} / U_{x}-t$ can thus be interpreted as a spatial variable $u(x)=x / U_{x}-t_{0}$

192 consecutive bubbles, separated by only two diameters, have been correctly switched before one error occurred. The efficiency collapses rapidly if the bubbles are closer. In this case, this very good efficiency, up to approximately 17 bubbles per second, was reached by applying the acoustic deviation to the bubbles with an activation time of $30 \mathrm{~ms}$ in the experiment of Fig. 9, just enough to push the bubble in the desired branch without disturbing the following one. This system works endlessly as long as the bubbles do not get closer than three diameters.

\section{Controlled asymmetric bubble}

We use a variant setup: it includes a global forcing because the PDMS is glued on a large glass slide that acts as a resonator. It is used at a fixed frequency $f=143 \mathrm{kHz}$, with a varying ultrasound amplitude, measured by the voltage applied to the transducer. When we increase the voltage from 0 to $30 \mathrm{~V}$, in a succession of 20 steps, the bubble breakup becomes increasingly asymmetric, with the larger of the two bubbles formed going into the left channel (see Fig. 10). For $f=147 \mathrm{kHz}$, the result is similar, but the larger bubble goes into the right branch. We thus easily produce populations of bubbles with two different controlled sizes.

\section{CONCLUSIONS}

The results show that the acoustic force applied at the resonance of the bubbles can be very strong, large enough to manipulate the bubbles in a microfluidic circuit. The model 
(a)

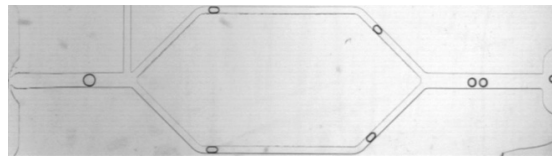

(b)

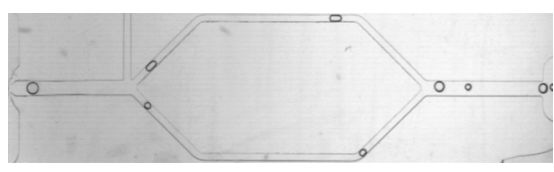

(c)

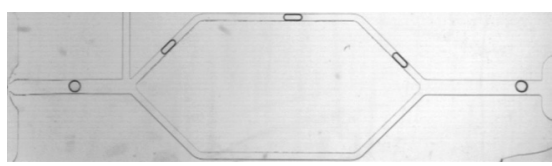

FIG. 10. Controlled asymmetric bubble breakup by increasing the ultrasound amplitude by steps from 0 to $30 \mathrm{~V}$. For increasing ultrasound amplitude we encounter: (a) $V=0 \mathrm{~V}$, unperturbed flow, bubble breakup in two equal parts, (b) intermediate $V$, asymmetric breakup, the largest part flows into the left branch, (c) $V=30 \mathrm{~V}$, no breakup, bubbles are entirely deviated on the left branch. The ultrasound frequency is set at $f=143 \mathrm{kHz}$, flow parameters: $Q_{l}=250 \mu 1 \mathrm{~min}^{-1}, P_{g}=3.90 \mathrm{kPa}$.

of the Bjerknes force has been extended to confined bubbles with a modification of the Minnaert relation. Perspectives include a better modeling of the bubble pulsation, taking into account the real 3D shape of the confined bubbles, which should provide a ground for the modified Minnaert law we introduced. On the side of applications, these gas samples provide a simple model system for the manipulation of soft and compressible objects, such as gaseous contrast agents or any gas filled biological material.

${ }^{1}$ J. P. Urbanski, W. Thies, C. Rhodes, S. Amarasinghe, and T. Thorsen, "Digital microfluidics using soft lithography," Lab Chip 6, 96 (2006).

${ }^{2}$ P. Garstecki, I. Gitlin, W. DiLuzio, G. M. Whitesides, E. Kumacheva, and H. A. Stone, "Formation of monodisperse bubbles in a microfluidic flowfocusing device," Appl. Phys. Lett. 85, 2649 (2004).

${ }^{3}$ B. Dollet, W. van Hoeve, J.-P. Raven, P. Marmottant, and M. Versluis, "Role of the channel geometry on the bubble pinch-off in flow-focusing devices," Phys. Rev. Lett. 100, 034504 (2008).

${ }^{4}$ H. A. Stone, A. D. Stroock, and A. Ajdari, "Engineering flows in small devices: Microfluidics toward a lab-on-a-chip," Annu. Rev. Fluid Mech. 36, 381 (2004).

${ }^{5}$ G. M. Whitesides, "The origins and the future of microfluidics," Nature (London) 442, 368 (2006).

${ }^{6}$ M. Hashimoto, S. S. Shevkoplyas, B. Zasońska, T. Szymborski, P. Garstecki, and G. M. Whitesides, "Formation of bubbles and droplets in parallel, coupled flow-focusing geometries," Small 4, 1795 (2008).

${ }^{7}$ J. Wan, A. Bick, M. Sullivan, and H. A. Stone, "Controllable microfluidic production of microbubbles in water-in-oil emulsions and the formation of porous microparticles," Adv. Mater. (Weinheim, Ger.) 20, 3314 (2008).

${ }^{8}$ T. G. Leighton, P. R. White, and M. A. Marsden, "The one-dimensional bubble: An unusual oscillator, with applications to human bioeffects of underwater sound," Eur. J. Phys. 16, 275 (1995).

${ }^{9}$ T. Lilliehorn, U. Simu, M. Nilsson, M. Almqvist, T. Stepinski, T. Laurell, J. Nilsson, and S. Johansson, "Trapping of microparticles in the near field of an ultrasonic transducer," Ultrasonics 43, 293 (2005).

${ }^{10}$ A. Haake, "Micromanipulation of small particles with ultrasound," Ph.D. thesis, Swiss Federal Institute of Technology Zurich, 2004.

${ }^{11}$ T. Laurell, F. Petersson, and A. Nilsson, "Chip integrated strategies for acoustic separation and manipulation of cells and particles," Chem. Soc. Rev. 36, 492 (2007).

${ }^{12}$ V. F. J. Bjerknes, Fields of Force (Columbia University Press, New York, 1906).

${ }^{13}$ T. G. Leighton, A. J. Walton, and M. J. W. Pickworth, "Primary bjerknes forces," Eur. J. Phys. 11, 47 (1990).

${ }^{14}$ T. G. Leighton, The Acoustic Bubble (Academic, London, 1994)

${ }^{15}$ R. Dangla and C. Poulain, "When sound slows down bubbles," Phys. Fluids 22, 041703 (2010).

${ }^{16}$ R. A. Millikan, "On the elementary electrical charge and the Avogadro constant," Phys. Rev. 2, 109 (1913).

${ }^{17}$ S. R. K. Maruvada and C.-W. Park, "Retarded motion of bubbles in heleshaw cells," Phys. Fluids 8, 3229 (1996).

${ }^{18}$ M. J. Fuerstman, A. Lai, M. E. Thurlow, S. S. Shevkoplyas, H. A. Stone, and G. M. Whitesides, "The pressure drop along rectangular microchannels containing bubbles," Lab Chip 7, 1479 (2007).

${ }^{19}$ D. Duffy, J. McDonald, O. Schueller, and G. Whitesides, "Rapid prototyping of microfluidic systems in poly(dimethylsiloxane)," Anal. Chem. 70, 4974 (1998).

${ }^{20}$ D. Rabaud, "Microbulles sous champ acoustique: Manipulation et interaction," Ph.D. thesis, University Joseph-Fourier, 2010.

${ }^{21}$ E. Lacot and O. Hugon, "Phase-sensitive laser detection by frequencyshifted optical feedback," Phys. Rev. A 70, 053824 (2004).

${ }^{22}$ G. Taylor and P. G. Saffman, "A note on the motion of bubbles in a hele-shaw cell and porous medium,” Q. J. Mech. Appl. Math. 12, 265 (1959).

${ }^{23}$ N. D. Denkov, V. Subramanian, D. Gurovich, and A. Lips, "Wall slip and viscous dissipation in sheared foams: Effect of surface mobility," Colloids Surf., A 263, 129 (2005).

${ }^{24}$ F. P. Bretherton, "The motion of long bubbles in tubes," J. Fluid Mech. 10, 166 (1961).

${ }^{25}$ J. Emile, A. Salonen, B. Dollet, and A. Saint-Jalmes, "A systematic and quantitative study of the link between foam slipping and interfacial viscoelasticity," Langmuir 25, 13412 (2009).

${ }^{26}$ R. Höhler and S. Cohen-Addad, "Rheology of liquid foam," J. Phys.: Condens. Matter 17, R1041 (2005).

${ }^{27}$ E. Terriac, J. Etrillard, and I. Cantat, "Viscous force exerted on a foam at a solid boundary: Influence of the liquid fraction and of the bubble size," Europhys. Lett. 74, 909 (2006).

${ }^{28}$ I. Cantat, N. Kern, and R. Delannay, "Dissipation in foam flowing through narrow channels," Europhys. Lett. 65, 726 (2004).

${ }^{29}$ A. Prosperetti, "Bubbles," Phys. Fluids 16, 1852 (2004).

${ }^{30}$ D. Fuard, T. Tzvetkova-Chevolleau, S. Decossas, P. Tracqui, and P. Schiavone, "Optimization of poly-di-methyl-siloxane (pdms) substrates for studying cellular adhesion and motility," Microelectron. Eng. 85, 1289 (2008).

${ }^{31}$ L. V. King, "On the acoustic radiation pressure on spheres," Proc. R. Soc. London, Ser. A 147, 212 (1934). 